\title{
Information Theory Based Evaluation of Interactions Between RR and QT Intervals in the Normal and People with High Risk for Cardiac Arrhythmias
}

\author{
Chenxi Li, Yue Pan, Ping Zhan, Zhigang Wang, Zhengguo Zhang, Yi Peng \\ Institute of Basic Medical Sciences, Chinese Academy of Medical Sciences \& School of Basic \\ Medicine, Peking Union Medical College, Beijing, China
}

\begin{abstract}
This project is purposed to evaluate autonomic nerve system (ANS) through the coupling of RR interval (RRI) and QT interval (QTI) using information theory as well as Spearman correlation coefficient (Spearman CC). And the possible changes of their coupling in ESRD (those with high risk for cardiac arrhythmias and sudden cardiac death).The Holter data with 189 Normal and 43 ESRD were provided by THEW. 5min episodes at rest were selected both in daytime and on night. Let predictive information (PI) measure the information that carried by QTI can be predicted by the knowledge of the past of itself and RRI. The information storage and internal information of $Q T$ variability $(Q T V)$ were determined respectively as the self-entropy of the QTI series and the self-entropy of the series conditioned to the knowledge of RRI. The information transfer and cross information from RRI to QTV were assessed as the coupling of them two. PI on night $\left(P I_{n}\right)$ was significantly smaller in ESRD compared with that in Normal $(0.258 \pm 0.137$ vs $0.322 \pm 0.142$ ). However, no significant difference was found between PI in daytime $\left(P I_{d}\right)$ in Normal and in ESRD. And the decreased PI ${ }_{n}$ led the loss of PI diurnal variation in $\operatorname{ESRD}(0.275 \pm 0.170$ vs $0.258 \pm 0.137)$. While in Normal, there were significant difference between $P I_{d}$ and $P I_{n} \quad(0.223 \pm 0.102$ vs $0.322 \pm 0.142)$. Since the decreased $P I_{n}$ in ESRD was mostly due to the changes in transfer entropy, meaning the reduction of RRI and QTI coupling. At the same time, the decreased conditioned self-entropy of QTI revealed less predictability of the past QTI to its present. All the above reflected the loss of sympathetic inhibition in ESRD, especially on night when the vagal activity is dominant and sympathetic activity should be withdrawn. The information theory based approach is not only feasible for assessing ANS activity through the coupling of RRI and QTI, but also provides a measure to characterize QTV related to HRV as well as those unrelated to HRV from nonlinear view.
\end{abstract}

\section{Introduction}

The modulation of autonomic nerve system (ANS) to sinus node results in the dynamic changes of RR interval (RRI) and heart rate variability (HRV). QT interval (QTI) is defined as the temporal difference between the QRS complex onset and the $\mathrm{T}$ wave end. In addition to its main dependency on RRI, QTI is directly influenced by ANS and other factors $[1,2]$. The coupling within and between RRI and QTI can be linear or nonlinear. However, this coupling can be changed by physiological factors (such as day and night alternation) and autonomic neuropathy caused by diseases, such as myocardial infarction, heart failure, diabetes, hypertension and so on [3-5].

In our previous study [6], we applied cross-spectrum method, measuring the linear coupling of RRI and QTI, to characterize alterations in ANS behaviors of those with high risk for cardiac arrhythmias and sudden cardiac death. We have shown that by directional correlation analysis of RRI and QTI based on transfer function. In the present study, an analysis of nonlinear coupling dynamics of RRI and QTI was conducted using information theory, which has proven to be useful to assess directional interactions between time series [7-9]. Information decomposition was used to assess information storage and internal information of QTV, and information transfer and cross information from HR to QTV. It is hypothesized that information theory based measures may reveal altered ANS behaviors with high risk for cardiac arrhythmias. Additionally, linear coupling of RRI and QTI is assessed by Spearman correlation coefficient (Spearman CC).

\section{Materials and methods}

The flow diagram of our study is presented in Fig1. The date for this analysis were based on Holter data of database Normal and ESRD in Telemetric and Holter ECG Warehouse (THEW, http://www.thew-project.org). 5 min episodes in resting state were selected from each Holter recording both in day and on night. RRI and QTI were extracted from each selected episode. For the extracted RRI and QTI, the mean and standard deviation of these interval series as well as the Spearman CC between the RRI and QTI were calculated. Let RRI as the driving process and QTI as the target process, an 
information decomposition method based on information theory was applied to selected RRI and QTI series to get predictive information of target process as well as indicators that reflect the internal dynamics of target process and interactions from driving process to target process. The relevant indices results were compared to verify whether there were statistical differences among the Normal and ESRD in terms of circadian rhythm.

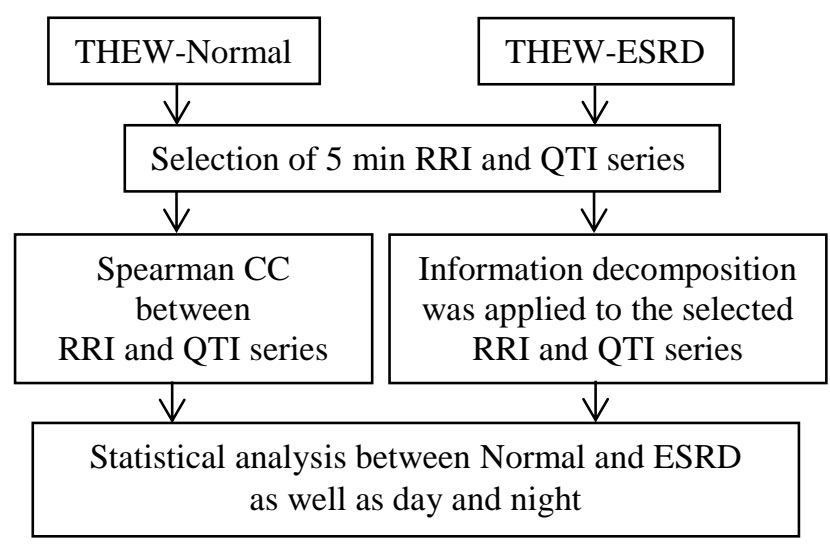

Fig1. The flow diagram of our study

\subsection{Data source}

In THEW, Database Normal (E-HOL-03-0202-003, ranging from 9 82 y) contains $24 \mathrm{~h}$ Holter recordings of 202 healthy subjects. The other database is ESRD (EHOL-12-0051-016, more than $40 \mathrm{y}$ ), including $48 \mathrm{~h}$ Holter recordings of 51 end stage renal disease patients with high risk for cardiac arrhythmias and sudden cardiac death. With the exclusion of those incomplete records, 189 records from Normal and 43 records from ESRD were finally selected.

For each selected recording, 2 episodes (each lasting $5 \mathrm{~min}$ ) in resting state were extracted, one in the period of 7:00 20:00(day), the other in 0:00 6:00 (night). RRIs and QTIs corresponding to the selected episodes were obtained using a complex algorithm validated with the records in Physionet QT Database (http://www.physionet.org/ physiobank/database/qtdb/)[10]. Ectopic beats were removed before further analysis.

\subsection{Information Decomposition}

Information theory has proven to be useful to assess directional interactions between cardiorespiratory time series [11]. Considering a system consisting of two interacting subsystems $X$ and $Y$, let $X$ as the driving process and $Y$ as the target process. Assuming that the evolution of the systems over time is described by the dynamic bivariate process $\{X, Y\}$. Setting a time reference point and $n$ is the present time. $X_{n}$ and $Y_{n}$ are the univariate variables describing the present of the processes $X$ and $Y$, and $X_{n}^{-}=\left[X_{n-1}, X_{n-2}, \cdots\right]$ and $Y_{n}^{-}=\left[Y_{n-1}\right.$, $\left.Y_{n-2}, \cdots\right]$ are the multivariate variables describing the past of the processes.

Then, the effect of the joint process $\{X, Y\}$ on the target process $Y$ is measured by the predictive information(PI):

$$
\mathrm{PI}_{Y}=H\left(Y_{n}\right)-H\left(Y_{n} \mid X_{n}^{-}, Y_{n}^{-}\right)
$$

Where $H\left(Y_{n}\right)=-\sum p\left(y_{n}\right) \ln p\left(y_{n}\right)$ is the Shannon entropy, and $H\left(Y_{n} \mid X_{n}^{-}, Y_{n}^{-}\right)$is conditional entropy. Predictive information $\mathrm{PI}_{Y}$ quantifies the part of the information carried by the present of the target process $Y$ that can be predicted by the past of the joint process $\left[X_{n}^{-}, Y_{n}^{-}\right]$, i.e. how much of the information carried by $Y_{n}$ can be predicted by the knowledge of the past of $X$ and $Y$.

In the framework of information dynamics, the temporal evolution of coupled systems can be studied by decomposing the predictive information about an assigned target system into amounts quantifying the information stored inside the system and the information transferred to it. Therefore, the prediction information $\mathrm{PI}_{Y}$ can be decomposed as:

$$
\mathrm{PI}_{Y}=H\left(Y_{n}\right)-H\left(Y_{n} \mid Y_{n}^{-}\right)+H\left(Y_{n} \mid Y_{n}^{-}\right)-H\left(Y_{n} \mid X_{n}^{-}, Y_{n}^{-}\right)
$$

The former two items $S_{Y}=H\left(Y_{n}\right)-H\left(Y_{n} \mid Y_{n}^{-}\right)$in the formula is the self-entropy, which is a measure of information storage, quantifying the part of the information carried by $Y_{n}$ that can be predicted by its own past. The latter two $T_{X \rightarrow Y}=H\left(Y_{n} \mid Y_{n}^{-}\right)-H\left(Y_{n} \mid X_{n}^{-}, Y_{n}^{-}\right)$is the transfer entropy, a measure of information transfer quantifying the part of the information carried by $Y_{n}$ that can be predicted by the past of $X$ on condition that $Y_{n}^{-}$is known. $T_{X \rightarrow Y}$ describes the additional predictability that the past of $X$ brings about the present of $Y$ that was not brought already by the past of $Y$.

Alternatively, the prediction information $\mathrm{PI}_{Y}$ can be decomposed by first considering the past of $X$ :

$$
\mathrm{PI}_{Y}=H\left(Y_{n}\right)-H\left(Y_{n} \mid X_{n}^{-}\right)+H\left(Y_{n} \mid X_{n}^{-}\right)-H\left(Y_{n} \mid X_{n}^{-}, Y_{n}^{-}\right)
$$

The former two items $C_{X \rightarrow Y}=H\left(Y_{n}\right)-H\left(Y_{n} \mid X_{n}^{-}\right)$in the formula is the cross-entropy, quantifying the part of the information carried by $Y_{n}$ that can be predicted by the past of $X$. The latter two $S_{Y \mid X}=H\left(Y_{n} \mid X_{n}^{-}\right)-H\left(Y_{n} \mid X_{n}^{-}, Y_{n}^{-}\right)$is the conditional self-entropy, quantifying the part of the information carried by $Y_{n}$ that can be predicted by its own past on condition that $X_{n}^{-}$is known. $S_{Y \mid X}$ describes the additional predictability that the past of $Y$ brings about the present of $Y$ that was not brought already by the past of $X$.

\subsection{Statistic analysis}

All indices are expressed as mean \pm standard deviation (SD). The Wilcoxon rank sum test was applied to detect 
differences between the Normal and ESRD and differences of the circadian indices in two groups.

The statistical analysis was performed using SPSS 22.0 (SPSS Inc, Chicago, USA). Statistical significance was accepted at the level of $P<0.05$.

\section{Results}

The results of our study are shown in Table 1. It could be seen from the time-domain indices that diurnal variation of RRI and QTI exists in both Normal and ESRD, and the value was significantly smaller at day. In addition, there were significant differences of almost all RRI and QTI between Normal and ESRD except for QTI at night. Specifically, RRI by day in Normal was smaller than that in ESRD while the situation is reversed for RRI at night.

The Spearman CC of RRI and QTI during daytime was significantly higher than that at night in Normal. For
ESRD, no diurnal variation of that was observed. And for this indice, there was significantly decreased in ESRD compared with Normal.

For the results of information decomposition, when RRI as the driving process and QTI as the target process, $\mathrm{PI}$ on night $\left(\mathrm{PI}_{\mathrm{n}}\right)$ was significantly smaller in ESRD compared with that in Normal $(0.258 \pm 0.137$ vs $0.322 \pm 0.142)$. However, no significant difference was found between PI in daytime $\left(\mathrm{PI}_{\mathrm{d}}\right)$ in Normal and in ESRD. While in Normal, there were significant difference between $\mathrm{PI}_{\mathrm{d}}$ and $\mathrm{PI}_{\mathrm{n}}(0.223 \pm 0.102$ vs $0.322 \pm 0.142)$. The self-entropy and the cross-entropy showed the same change as the PI between the two groups. PI was significantly increased from day to night in Normal, but this diurnal variation did not exist in ESRD. The decreased $\mathrm{PI}_{\mathrm{n}}$ led the loss of PI diurnal variation in ESRD $(0.275 \pm 0.170$ vs $0.258 \pm 0.137)$. Several entropy measures of information decomposition had the same diurnal variation as that of PI.

Table 1 The analysis results in Normal and ESRD

\begin{tabular}{|c|c|c|c|c|}
\hline \multirow{2}{*}{ Index } & \multicolumn{2}{|c|}{ Normal $(n=189)$} & \multicolumn{2}{|c|}{ ESRD $(n=43)$} \\
\hline & Day & Night & Day & Night \\
\hline RRI (ms) & $733 \pm 113^{* \#}$ & $948 \pm 154^{\#}$ & $799 \pm 119 *$ & $869 \pm 123$ \\
\hline QTI (ms) & $371 \pm 27 *^{\#}$ & $419 \pm 33$ & $403 \pm 40 *$ & $423 \pm 40$ \\
\hline$\rho_{\mathrm{RRI}, \mathrm{QTI}}$ & $0.264 \pm 0.168 * \#$ & $0.179 \pm 0.144$ & $0.206 \pm 0.256$ & $0.129 \pm 0.179$ \\
\hline$S_{\mathrm{QTI}}$ & $0.061 \pm 0.042 *$ & $0.090 \pm 0.072^{\#}$ & $0.097 \pm 0.104$ & $0.067 \pm 0.050$ \\
\hline$T_{\mathrm{RRI} \rightarrow \mathrm{QTI}}$ & $0.162 \pm 0.076^{*}$ & $0.232 \pm 0.105^{\#}$ & $0.178 \pm 0.110$ & $0.191 \pm 0.119$ \\
\hline$C_{\mathrm{RRI} \rightarrow \mathrm{QTI}}$ & $0.083 \pm 0.049^{*}$ & $0.098 \pm 0.063$ & $0.117 \pm 0.118$ & $0.084 \pm 0.054$ \\
\hline$S_{\mathrm{QTI} \mid \mathrm{RRI}}$ & $0.139 \pm 0.079 *$ & $0.224 \pm 0.115^{\#}$ & $0.159 \pm 0.100$ & $0.174 \pm 0.120$ \\
\hline$P I_{\mathrm{QTI}}$ & $0.223 \pm 0.102 *$ & $0.322 \pm 0.142^{\#}$ & $0.275 \pm 0.170$ & $0.258 \pm 0.137$ \\
\hline
\end{tabular}

Note: Data are expressed as mean $\pm \mathrm{SD} ;{ }^{*} P<0.05$ Day $v s$ Night within a group; ${ }^{\#} P<0.05$ Normal $v s$ ESRD during the same period of one day.

\section{Discussion}

Based on the Spearman $\mathrm{CC}$ and information decomposition, we investigated the coupling of RRI and QTI from linear and nonlinear aspects. Differences were revealed between Normal and ESRD concerning their ANS behaviors. Significant circadian differences existed in all indexes both in Normal, but were lost in ESRD for both Spearman CC and PI.

In the study of Seethala et al [12], they sought to assess $\beta$-adrenergic stimulation effects on QTI response to HR (or RRI) change with normal subjects (except for supraventricular tachycardia). They described QTI response to RRI change as two distinct phases, an immediate response (IR) followed by a gradual monoexponential course to the new steady state value. And the IR is based on the assumption of an approximately linear relationship between immediate changes of QTI to RRI. Their results showed that $\beta$ adrenergic stimulation increased the immediate QT response to RRI change, that is the enhanced sympathetic activity might increase IR magnitude of RRI change. Our results are in accordance with the above ones. In Normal, the Spearman CC of QTI and RRI in daytime is significantly larger than that on night due to higher sympathetic activity in daytime. As for ESRD, though there is the tendency for Spearman CC to decrease from day to night, no significant difference emerges. In ESRD, though RRI on night is obviously smaller than that in Normal, the QTIs of them two are almost at the same level. The prolonged QTI in ESRD reflects insufficient withdrawal of sympathetic activity on night.

In contrast to the reduction of Spearman $\mathrm{CC}$ in night in Normal, PI increased on night compared with that in daytime. The significant increase in PI means the better prediction of QTI, or the less variability of QTI (QTV) [13].While in ESRD, the PIs in daytime and on night are almost at the same level. From linear and nonlinear aspects, we found different coupling behaviors of RRI and QTI about circadian rhythm in normal people and those with high risk for cardiac arrhythmias.

In this study, information dynamics was used to assess information storage and internal information of QTV, and information transfer and cross information from heart rate to QTV. Furthermore, the composition of QTV could be 
obtained upon this kind of analysis. Transfer entropy in Equation (2) measures the information transferring from the driver to the target process and will always be zero in the absence of a causal link from driver to target. Here, $T_{\mathrm{RRI} \rightarrow \mathrm{QTI}}$ represents the QTV that is related to HRV. On the other hand, the conditional self-entropy in Equation (3) reflects the internal information in the target process because it will always be zero in the absence of internal dynamics in the target, and it is not influenced by the dynamics of the driver process. So, $S_{\text {QTIRRI }}$ indicates the QTV that is unrelated to HRV. Not only the interaction between sympathetic modulation and the coupling of HRV and QTV, but also the influence of sympathetic modulation on ventricular repolarization, can be interpreted by information decomposition analysis. For Normal, increased PI (more predictive information) for QTI on night means the decrease in QTV that is in line with situation represented by QTVi in Ref [14]. Furthermore, the decreased $\mathrm{PI}_{\mathrm{n}}$ in ESRD was mostly due to the changes in transfer entropy, meaning the reduction of RRI and QTI coupling. At the same time, the decreased conditioned self-entropy of QTI revealed less predictability of the past QTI to its present. All the above reflected the loss of sympathetic inhibition in ESRD, especially on night when the vagal activity is dominant and sympathetic activity should be withdrawn.

\section{Conclusion}

The information theory based approach is feasible for assessing ANS activity through the coupling of RRI and QTI. Moreover, this method provides a measure to characterize QTV related to HRV as well as those unrelated to HRV from nonlinear view.

\section{Acknowledgements}

This work received financial support from the National Natural Science Foundation of China through the project 81471746 and 81071225.

\section{References}

[1] Ahnve S, Vallin H.Influence of heart rate and inhibition of autonomic tone on the QT interval. Circulation1982; 65(3): 435-9.

[2] Gastaldelli A, Emdin M, Conforti F, et al. Insulin prolongs the QTc interval in humans. Am J Physiol Regul Integr
Comp Physiol 2000; 279: R2022-5.

[3] Porta A, Tobaldini E, Gnecchi-Ruscone T, et al. RT variability unrelated to heart period and respiration progressively increases during graded head-up tilt. Am J Physiol Heart Circ Physiol 2010; 298:H1406-20.

[4] Baumert M, Schlaich MP, Nalivaiko E, et al. Relation between QT interval variability and cardiac sympathetic activity in hypertension. Am J Physiol Heart Circ Physiol 2011; 300: 1412-7.

[5] Nahahoni E, Strasberg B, Adler E, et al. Complexity of the dynamic QT variability and RR variability in patients with acute anterior wall myocardial infarction. J Electrocardiology 2004; 37(3): 173-9.

[6] Zhan P, Li C, Liu H, et al. Reflection of autonomic regulation behavior using short-term cross-spectral analysis on RR and QT intervals. Computing in Cardiology 2016; 43: 154-7.

[7] Faes L, Porta A, Nollo G. Information decomposition in bivariate systems: Theory and application to cardiorespiratory dynamics. Entropy 2015; 17:277-303.

[8] Faes L, Nollo G, Porta A. Information domain approach to the investigation of cardio-vascular, cardiopulmonary, and vasculo-pulmonary causal couplings. Front Physiol 2011; 2:80.

[9] Faes L, Nollo G, Porta A. Non-uniform multivariate embedding to assess the information transfer in cardiovascular and cardiorespiratory variability series. Comput Biol Med 2012; 42:290-7.

[10] Sun ZW, Peng Y. Study on a complex algorithm for detecting QT interval. Biomedical Engineering and Clinical Medicine2009; 13: 184-8.

[11] Widjaja D, Montalto A, Vlemincx E, et al. Cardiorespiratory information dynamics during mental arithmetic and sustained attention. PLoS ONE 2015; 10(6): $\mathrm{e} 0129112$.

[12] Seethala S, Shusterman V, Saba S, et al. Effect of $\beta$ adrenergic stimulation on QT interval accommodation. Heart Rhythm 2011; 8: 263-70.

[13] Visnovcova Z, Mestanik M, Javorka M, et al. Complexity and time asymmetry of heart rate variability are altered in acute mental stress[J]. Physiol Meas 2014; 35(7):1319-34.

[14] Zhu Y, Yang X, Wang Z, et al. An evaluating method for autonomic nerve activity by means of estimating the consistency of heart rate variability and QT variability. IEEE Transactions on Biomedical Engineering 2014; 61(3): 938-45.

Corresponding to: Yi Peng

Address: Department of Biomedical Engineering Institute of Basic Medical Sciences, CAMS

5 Dong Dan San Tiao

Beijing 100005, China

E-mail: pengyi@pumc.edu.cn 